University of Wollongong

Research Online

Faculty of Science, Medicine and Health -

Papers: Part B

Faculty of Science, Medicine and Health

2020

\title{
Safety and Efficacy of Using Nuts to Improve Bowel Health in Hemodialysis Patients
}

\author{
Kelly Lambert \\ University of Wollongong, klambert@uow.edu.au \\ Luke Bird \\ Addison Borst \\ University of Wollongong \\ Andrew Fuller \\ University of Wollongong \\ Yanan Wang
}

See next page for additional authors

Follow this and additional works at: https://ro.uow.edu.au/smhpapers1

\section{Publication Details Citation}

Lambert, K., Bird, L., Borst, A., Fuller, A., Wang, Y., Rogers, G., Stanford, J., Sanderson-Smith, M. L., Williams, J., McWhinney, B., Neale, E., Probst, Y., \& Lonergan, M. A. (2020). Safety and Efficacy of Using Nuts to Improve Bowel Health in Hemodialysis Patients. Faculty of Science, Medicine and Health - Papers: Part B. Retrieved from https://ro.uow.edu.au/smhpapers1/1200 


\title{
Safety and Efficacy of Using Nuts to Improve Bowel Health in Hemodialysis Patients
}

\author{
Abstract \\ Objective: Constipation is common in patients with end-stage kidney disease. Nondrug strategies to \\ manage constipation are challenging because of dietary potassium, phosphate, and fluid restrictions. \\ Nuts are a high-fiber food but are excluded from the diet because of the high potassium and phosphate \\ content. The aim of this study was to examine the safety and efficacy of using nuts to improve \\ constipation in adults undertaking hemodialysis (HD). Design and Methods: Adult patients undertaking \\ $\mathrm{HD}$ were recruited to this nonrandomized, 10 -week repeated measures, within-subject, pragmatic clinical \\ trial, conducted in two HD units. The intervention consisted of consumption of $40 \mathrm{~g}$ of raw almonds daily \\ for four weeks, followed by a two-week washout and four-week control period. The primary safety \\ outcome measures were change in predialysis serum potassium and phosphate levels. The primary \\ efficacy outcome was reduction in constipation, measured using the Bristol Stool Form Scale and \\ Palliative Care Outcome Scale (POS-S) renal symptom score. Secondary outcomes included quality of life, \\ selected uremic toxins, cognition, gut microbiota profile, and symptom burden. Results: Twenty patients \\ completed the trial (median age: 67 [interquartile range: $57.5-77.8$ ] years, 51\% male). After controlling for \\ dialysis adequacy, anuria, dietary intake, bicarbonate, and parathyroid hormone, there were no statistically \\ significant changes in serum potassium $(P=0.21)$ or phosphate $(P=0.16)$ associated with daily \\ consumption of almonds. However, statistically significant improvements in constipation were seen at \\ weeks $2,3,4$, and 10 . There were statistically significant improvements in quality of life $(P=0.030)$, \\ overall symptom burden $(P=0.002)$, vomiting $(P=0.020)$, itching $(P=0.006)$, and skin changes $(P=$ \\ 0.002). Conclusion: Daily consumption of almonds for four weeks was safe, effective, and well tolerated. \\ Improvements in quality of life and symptom burden warrant further research to elucidate potential \\ mechanisms. The findings support the potential reinclusion of foods such as nuts into the diet of patients \\ who underwent HD.

\section{Publication Details} \\ Lambert, K., Bird, L., Borst, A., Fuller, A., Wang, Y., Rogers, G., Stanford, J., Sanderson-Smith, M., Williams, \\ J., McWhinney, B., Neale, E., Probst, Y. \& Lonergan, M. (2020). Safety and Efficacy of Using Nuts to \\ Improve Bowel Health in Hemodialysis Patients. Journal of Renal Nutrition.

\section{Authors} \\ Kelly Lambert, Luke Bird, Addison Borst, Andrew Fuller, Yanan Wang, Geraint Rogers, Jordan Stanford, \\ Martina L. Sanderson-Smith, Jonathan Williams, Brett McWhinney, Elizabeth Neale, Yasmine Probst, and \\ Maureen A. Lonergan
}


1 Title: Safety and efficacy of using nuts to improve bowel health in hemodialysis patients

\section{Abstract:}

\section{Objective:}

4 Constipation is common in patients with end stage kidney disease (ESKD). Non-drug

5 strategies to manage constipation are challenging due to dietary potassium, phosphate and

6 fluid restrictions. Nuts are a high fiber food but are excluded from the diet due to the high

7 potassium and phosphate content. The aim of this study was to examine the safety and

8 efficacy of using nuts to improve constipation in adults undertaking hemodialysis (HD).

\section{Design and methods:}

10 Adult patients undertaking HD were recruited to this non-randomised, 10-week repeated

11 measures, within subject, pragmatic clinical trial, conducted in two HD units. The

12 intervention period consisted of consumption of $40 \mathrm{~g}$ raw almonds daily for four weeks,

13 followed by a two-week washout and four-week control period. The primary safety outcome

14 measures were change in predialysis serum potassium and phosphate levels, and the primary

15 efficacy outcome was reduction in constipation, measured using the Bristol Stool Form Scale

16 and Palliative Care Outcome Scale (POS-S) renal symptom score. Secondary outcomes included

17 quality of life, selected uremic toxins, cognition, gut microbiota profile, and symptom

18 burden.

19 Results: 
1 Twenty patients completed the trial (median age 67 (IQR: 57.5-77.8) years, 51\% male). After

2 controlling for dialysis adequacy, anuria, dietary intake, bicarbonate, and parathyroid

3 hormone there were no statistically significant changes in serum potassium $(p=0.21)$ or

4 phosphate $(\mathrm{p}=0.16)$ associated with the daily consumption of almonds. However, statistically

5 significant improvements in constipation were seen at weeks 2, 3, 4 and 10. There were

6 statistically significant improvements in quality of life $(p=0.030)$, overall symptom burden

$7 \quad(p=0.002)$, vomiting $(p=0.020)$, itching $(p=0.006)$ and skin changes $(p=0.002)$.

\section{Conclusion:}

9 Daily consumption of almonds for four weeks was found to be safe, effective and well

10 tolerated. Improvements in quality of life and symptom burden warrant further research to

11 elucidate potential mechanisms. The findings support the potential re-inclusion of foods such

12 as nuts into the diet of haemodialysis patients.

13 Keywords: hemodialysis, nuts, clinical trial, treatment outcome, constipation 
2 Abnormal bowel health is common in patients with end stage kidney disease (ESKD), and is

3 characterised by impaired motility ${ }^{1}$, and symptoms such as abdominal pain, indigestion,

4 reflux and constipation ${ }^{2}$. Constipation is estimated to affect more than three quarters of

5 hemodialysis patients ${ }^{3}$. In addition to reduced quality of life (QOL) ${ }^{4}$, constipation also

6 contributes to hyperkalaemia ${ }^{5}$, and abdominal discomfort ${ }^{3}$. In patients undertaking

7 peritoneal dialysis, constipation can also cause catheter dislodgment ${ }^{6,7}$. Constipation has also

8 been identified as an outcome of concern by patients and carers ${ }^{8}$.

Poorly designed renal diet prescriptions for patients with ESKD can worsen constipation ${ }^{9,10}$

due to overzealous restriction of fruits, vegetables, legumes, nuts and whole grain cereal products. Typical strategies suggested to manage constipation in healthy populations such as increasing physical activity, fiber and fluid intake ${ }^{11}$ are particularly challenging for those with ESKD. This is due partly to the dietary phosphate, fluid and potassium restrictions that are required.

17 In recent years several studies have challenged the paradigm that some nutrient dense foods that are typically restricted in the renal diet may not need to be. For example, the bioavailability of phosphorus from plant-based food sources appears to be significantly lower than the phosphorus in animal derived food sources and phosphate additives ${ }^{12}$. Similarly, the bioavailability of potassium in diets rich in fruit, vegetables and legumes has been shown to be less than was previously predicted ${ }^{13,14}$. Studies such as these, in addition to the recent evidence of reduced mortality in patients following healthy diet patterns in Chronic Kidney 
1 Disease $(\mathrm{CKD})^{15,16}$, have led to suggestions that liberalisation of the renal dietary restrictions

2 for patients with ESKD may be warranted ${ }^{17}$.

4 Given the extent of bowel health problems such as constipation, and poor medication

5 compliance in patients undertaking dialysis ${ }^{18-20}$, alternative non-drug strategies to improve

6 constipation in hemodialysis patients are needed. Nuts may be one potential option to

7 improve the management of constipation. Nuts are high in protein, fiber, micronutrients and

8 polyunsaturated fatty acids ${ }^{21}$. However, they are also high in potassium and phosphate and as

9 result, are typically not recommended for inclusion in the diet of a patient who is undertaking hemodialysis. However, large epidemiological studies have demonstrated that regular nut consumption is associated with improved laxation in healthy populations ${ }^{22}$, as well as improved gut microbiota profile ${ }^{23}$, increased vascular health improved cognition function ${ }^{24}$ and reduced mortality ${ }^{25}$. A recent meta-analysis reported that a dietary pattern high in fruits, vegetables, legumes and nuts was associated with a reduced risk of death ${ }^{15}$.

Despite the benefits of nut consumption in the general population ${ }^{21}$, whether regular nut consumption is safe and effective at improving bowel habits in patients undertaking hemodialysis has not been explored. Given these knowledge gaps, the overall aim of this study was to determine the safety and efficacy of using a non-drug treatment in the form of almonds to improve bowel health in adult haemodialysis patients. The hypotheses of this trial were that consumption of $40 \mathrm{~g}$ of almonds daily for four weeks is safe and would result in an improvement in bowel health and specifically a reduction in the prevalence of constipation. As a secondary outcome, we hypothesised that the intervention would result in improvements in symptom burden, QOL, uremic toxins, cognition and gut microbiota profile. 


\section{Methods}

$2 \quad$ Study design and study population

3 A non-randomised 10-week repeated measures, within subject, pragmatic clinical trial was

4 conducted and is reported in accordance with the guidelines for transparent reporting of

5 studies with non-randomized designs ${ }^{26}$, and the checklist for intervention description and

6 replication ${ }^{27}$. The clinical trial was registered with the Australian New Zealand Clinical

7 Trials Registry (Registration number ACTRN 617000600347p). The joint University of

8 Wollongong Illawarra Shoalhaven Human Research Ethics Committee approved the study

9 (HE2017/332) and all study procedures were followed in accordance with the Declaration of

10 Helsinki.

12 The trial setting was two satellite haemodialysis units in a regional health district of New

13 South Wales, Australia. The trial population consisted of haemodialysis patients attending the

14 units for therapy. Exclusion criteria were individuals who were pregnant; allergic to almonds;

15 suffered from dysphagia; or had dental problems that would prohibit consumption of nuts.

16 Those with a current diagnosis of acute diverticulitis or faecal impaction and those deemed

17 by nursing or medical staff as unable to follow instructions adequately due to language or

18 cognitive impairment were also excluded from the trial.

Recruitment

21 All hemodialysis patients were approached while undertaking hemodialysis by a member of

22 the research team. No treating health professionals on the research team assisted with 
1 recruitment. Interested patients provided written informed consent prior to commencing the

2 trial

Study procedures

5 All assessments were conducted with participants during the second hour of their first thrice

6 weekly hemodialysis therapy sessions. Following two weeks of pre-intervention assessments,

7 participants were required to consume the intervention food for a four-week period, followed

8 by a two-week wash-out period; followed by a subsequent four-week control period.

\section{Intervention}

11 The intervention consisted of the consumption of $40 \mathrm{~g}$ raw unsalted almonds with skin daily

12 for four weeks. This equates to approximately 27 almonds per day. A schematic of the study

13 is shown in the Supplemental material, Figure 1. Almonds were chosen due to the small

14 volume required to supply $8 \mathrm{~g}$ of protein and at least $3 \mathrm{~g}$ of fiber. This volume of almonds also supplied $210 \mathrm{mg}$ of phosphate and $318 \mathrm{mg}$ of potassium ${ }^{28}$. According to renal diet specifications this quantity of almonds was considered to be a high phosphate and high potassium food source ${ }^{29}$. Other nuts such as walnuts or macadamias were not considered for the study as they required daily consumption in excess of $60 \mathrm{~g}$ which was considered to be a deterrent to optimal intake. A one-week supply of almonds was provided to all participants at the start of each trial week. All other components of their usual renal dietary restrictions were maintained (i.e. the diet continued to be fluid, potassium, phosphate, and sodium restricted). Participants were instructed to consume the intervention ad libitum but not exceed the $40 \mathrm{~g}$ almonds within a 24-hour period. A 40g scoop was provided to all participants to ensure an accurate and consistent dose was consumed daily. To ensure intervention fidelity, participants 
1 were instructed to return any remaining amounts of almonds each week prior to provision of

2 the following week's supply. Residual almond quantities were weighed to ascertain average consumption of the intervention each week and adherence with the study protocol. During the

$4 \quad$ wash out and control weeks, participants were instructed to avoid all nut and nut products as

5 per usual renal diet recommendations and to maintain their usual renal dietary prescription.

\section{$7 \quad$ Primary outcome measures}

8 The primary safety outcome measure of the trial was change in predialysis serum potassium 9 and phosphate levels. All pathology measures were taken on the first dialysis session of each week following the long dialysis break. Hyperkalaemia was defined as serum potassium $\geq 6$ $\mathrm{mmol} / \mathrm{L}$. Hyperphosphatemia was defined as serum phosphate $\geq 5.74 \mathrm{mg} / \mathrm{dL}$ (or $\geq 1.85$ $\mathrm{mmol} / \mathrm{L}$.)

14 The primary efficacy measure was change in bowel habits. Three measures of bowel health were utilised, based on previous research that demonstrated self-report of bowel habit to be highly variable using different tools ${ }^{9}$. Participants rated their bowel habits at baseline, weeks

$171-4,7$ and 10 using the Bristol Stool Form Scale with a score of 1 or 2 indicative of constipation ${ }^{30}$. Participants rated their bowel health using a bowel management algorithm ${ }^{9}$ which includes questions from the Rome III criteria for the diagnosis of constipation. Participants also self-rated their bowels using the Patient Outcome Scale- Renal ${ }^{31}$. This tool

21 lists 18 symptoms including weakness, nausea, vomiting, and constipation, and patients rate the severity and presence of the symptoms. 
2 The secondary outcomes of the trial included QOL, serum total and free concentrations of the

3 uremic toxins p-Cresyl Sulfate (PCS) and Indoxyl Sulfate (IS), stool microbiota, cognition

4 and symptom burden. The methods used to measure and collect these outcomes are described 5 in the Supplemental material.

\section{$7 \quad$ Other measures}

8 Nutrition assessment was completed by a trained dietitian using a validated nutrition

9 assessment tool for use with hemodialysis patients (the Patient Generated Subjective Global

10 Assessment ${ }^{32}$ ). This assessment was completed at baseline, week 4, 7, 10 of the trial. Dietary

11 intake during the study was recorded using a three-day food record completed by the patient

12 (or carer) including one weekend day, dialysis day and non-dialysis day. This was completed

13 at baseline, weeks $1-4,7,10$ of the trial. A diet history interview ${ }^{33}$ was also obtained from

14 the particpants by the same trained dietitian. This information was obtained at baseline,

15 weeks 4, 7 and 10. Quantitative analysis of dietary intake was undertaken using FoodWorks

16 (version 9, Xyris Pty Ltd, Highgate Hill, Queensland, Australia), using the AUSNUT 2011-

1713 food composition database ${ }^{28}$. All nutrient data was presented as mean (SD). All prescribed and over the counter medications, supplements or herbal preparations taken by the participants to assist with bowel health were recorded at the commencement of each week of the trial. This was confirmed by having patients bring in pill bottles and/or prescription lists to the research team for verification. 
1 An adverse event was defined as any fatal or life-threatening event, or an event posing a

2 significant hazard, or causing a side effect, and any experiences requiring hospitalisation.

3 Events were monitored and those occurring during the trial were discussed, documented and

4 actioned by the clinical team.

\section{$6 \quad$ Statistical analyses}

7 Normality of variables was assessed using the Shapiro Wilk test. Data is reported as median and interquartile range or mean and standard deviation where appropriate. All analyses were conducted using SPSS (version 25, IMB Corporation, Chicago, IL, USA). General linear models for repeated measures were tested using a heterogeneous compound symmetry structure as the variance at each time point were assumed not to be constant. These models were used to determine the change in serum potassium and phosphate after controlling for dialysis adequacy, dietary intake of potassium or phosphate, urine output, serum bicarbonate and serum PTH level. Analysis of the change in uremic toxins after controlling for anuria and dialysis adequacy was also conducted. The McNemar test was used to determine changes in the proportion with hyperkalaemia, hyperphosphatemia, adequate bowel habit and laxative use. The Friedman test on one way ANOVA for repeated measures was used to assess the change in QOL, uremic toxins, and cognition. Per protocol analyses are reported and a pvalue of 0.05 was considered statistically significant.

\section{Results}

22 Thirty two patients were recruited to the study, and 20 participants completed the study

23 (Figure 2). More than half of the participants were male, with a median age of 67.5 (IQR: 
1 the PG-SGA; almost half (45\%) were anuric (defined as less than 100mL urine per day), and

2 almost half (45\%) were deemed to be cognitively impaired at baseline on formal assessment.

3 Multi-morbidity was evident with diabetes, coronary artery and peripheral vascular disease

4 common. Laxative use was common, with $65 \%$ taking laxatives or aperients at baseline.

5 When compared to evidence based guidelines for the management of hemodialysis patients,

6 baseline dietary intake was considered to be inadequate for energy, protein, phosphate and

7 fiber ${ }^{34}$. Only $15 \%$ of participants reported consuming an adequate intake of dietary fiber $8 \quad(>25 \mathrm{~g} /$ day $)$.

Assessment of safety

11 Consumption of $40 \mathrm{~g}$ almonds daily for 4 weeks was not associated with increases in serum potassium (Table $2, \mathrm{p}=0.21$ ) or serum phosphate (Table $2, \mathrm{p}=0.16$ ) after controlling for confounders. The proportion of participants who were considered hyperkalemic or hyperphosphatemic also did not change. There was one adverse event during the trial related to the intervention which consisted of a patient experiencing a choking episode. No further medical assistance was required.

\section{Assessment of efficacy}

As shown in Table 2, compared to baseline, there was a significant reduction in the proportion of participants who reported constipation at weeks 2,3 and $10(p=0.04, p=0.006$ and $p=0.03$, respectively). The mean POS renal score for constipation also reduced significantly during the study $(\mathrm{p}=0.02)$. Laxative use declined significantly between baseline and week 4 of the intervention from $65 \%$ to $20 \%(p=0.02)$. The mean individual 
1 consumption of almonds during the intervention weeks indicated that adherence to the

2 intervention was high (Table 2, mean: 89\%).

Secondary outcomes

5 There was a significant improvement in QOL over the 10 weeks (Table 3). The EQ-5D-5L

6 index score increased by 0.15 quality-adjusted life-years (QALY) from baseline by week 10

$7 \quad(p=0.03)$. There was no change in mean EQ-5D-5L Visual Analogue Scale score, uremic

8 toxins, or cognition. However, total symptom burden decreased significantly from a mean

9 score at baseline of $16.7 \pm 6.7$ to $10.2 \pm 6.0$ by week 4 and $11.3 \pm 9.0$ by week $10(p=0.002)$.

10 Other significant changes in symptom scores occurred for vomiting $(p=0.02)$, constipation

$11(p=0.02)$, itching $(p=0.006)$ and skin changes $(p=0.002)$.

No differences were observed in faecal microbiota diversity (alpha- or beta-diversity), either before or following the intervention. The consumption of $40 \mathrm{~g}$ daily of almonds over 4 weeks did not have a measurable effect on faecal microbiota composition (the relative abundance of specific bacterial taxa) in individuals undertaking hemodialysis.

\section{Discussion}

In this safety and efficacy trial, consumption of $40 \mathrm{~g}$ almond daily for four weeks was not associated with harmful elevations of serum potassium or phosphate, or other major adverse events. The intervention was associated with significant improvements in constipation when measured using two separate patient reported outcome tools. This trial also saw significant improvements in QOL and symptoms such as vomiting, itch and dry skin during the 
1 intervention period. This non-drug intervention was also well tolerated by participants, with

2 excellent adherence to guidelines for consumption of the almonds provided.

4 Given the high prevalence of constipation among dialysis patients, safe and effective management methods are required. This trial suggests that regular nut consumption may be a potentially effective and safe non-drug alternative or adjunct to traditional methods used to manage constipation. The effect size of the reduction in constipation between baseline and week 4 was equivalent to a moderately large effect (Cohen's $d 0.58$ ) and has obvious

9 practical importance ${ }^{35}$. Physiological mechanisms for how nuts may assist with laxation appear to be more than just the provision of the additional fiber. For example, some authors suggest the effect on laxation may be related to the presence of large amounts of fermentable carbohydrates including xylose and galactose in the almond cell wall. These carbohydrates are known to exert a prebiotic effect ${ }^{36}$, providing the substrate for bacterial metabolism that may confer a benefit for the human host ${ }^{37}$. Others suggest that nut digestion induces remodelling of the gut microbiota ${ }^{38}$ via improved butyrate synthesis from the metabolism of the fiber and polyphenol content.

In addition to constipation, patients undergoing dialysis commonly report dermatological symptoms. Mechanisms for the improved skin and reduction in itch (pruritus) reported by participants in our trial are unknown. Pruritus and xerosis (dry skin) in dialysis patients are understudied ${ }^{39}$, despite pruritus being experienced by almost half of dialysis patients. These complaints are also associated with reduction in the QOL of dialysis patients ${ }^{40}$. Traditionally, it is thought that xerosis is due to a reduction in the eccrine sweat glands and atrophy of sebaceous glands ${ }^{41}$. It is unclear what role diet may have in these conditions. However, one 
1 may speculate that the improvements in skin and pruritus may be due to the high

2 monounsaturated fat and vitamin E composition of the almonds. Vitamin E intake has been

3 associated with skin quality ${ }^{42}$, and dietary intake of vitamin $\mathrm{E}$ is reduced in individuals with

4 end stage kidney disease due to a reduction in consumption of rich food sources (nuts,

5 spinach and wholegrain cereals). Metabolism of vitamin E is also impaired in dialysis

6 patients ${ }^{43}$. Future research to examine the relationship between skin and dietary quality is

7 warranted.

9 This trial adds to the evidence base regarding the high symptom burden and poor QOL amongst dialysis patients ${ }^{44}$, especially those with constipation ${ }^{4}$. In this trial, the participants experienced a range of symptoms in addition to constipation, and the QOL was far below reported Australian norms (mean index score 0.91 and mean VAS score 78.55) even at baseline ${ }^{45}$. The reasons for the gain of 0.15 of a QALY during the trial period are unknown. Qualitative research to ascertain why this occurred would have been beneficial and could be an area for future research.

17 There are several strengths to this study. This includes the comprehensive collection of data regarding potential confounders to the results including in depth dietary data. However, there are also several obvious limitations. These include the small sample size and short follow up period. Unlike drug studies, it is difficult to blind participants to whole food interventions, thus, it is also possible that participants may have been subject to reporting bias. We used standard tools to assess efficacy outcomes, however, it should be noted that these tools rely on self-reported changes in bowel habits, which may have also introduced bias. Given the free-living nature of the participants, this challenge is likely to persist in future studies. We 
1 attempted to minimise inter-individual variation by using a study design where participants

2 acted as their own controls and undertook the intervention first. This was due to concerns regarding compliance and potential contamination of the intervention. It was also not considered appropriate to randomise participants in each site to start on either the intervention or control period and practical challenges coordinating between sites prevented randomisation by shift. As a result, we cannot conclude that bias may have influenced the study findings. It should be noted that many of the improvements seen during the intervention period (for example frequency of bowel movements, use of laxatives) were mitigated during the control period, although they remained more favourable than baseline levels. These results suggest that the intervention was partly responsible, although there may have been some continued carry-over effects observed during the control period.

To conclude, this trial offers insights into the utility of including nuts in the diet of haemodialysis patients to improve bowel health. In addition to being a safe, effective and well tolerated treatment, this trial also found there were improvements in QOL, and symptoms such as vomiting, itching and skin changes. Future research into the use of foods such as nuts to assist with constipation are needed, especially studies with a more robust study design. Despite no known mechanism, studies exploring the relationship between dietary changes and skin quality and pruritus will be beneficial for developing potential treatments. Additional research to elucidate potential mechanisms for these findings is warranted. Further exploration of dietary patterns in those undertaking dialysis, especially in those with suboptimal bowel health may also be useful. 
of haemodialysis patients.

\section{References}

1. Grant CJ, Harrison LE, Hoad CL, Marciani L, Gowland PA, Mclntyre CW. Patients with chronic kidney disease have abnormal upper gastro-intestinal tract digestive function: A study of uremic enteropathy. J Gastroenterol Hepatol. 2017;32(2):372-377.

2. Zuvela J, Trimingham C, Le Leu R, et al. Gastrointestinal symptoms in patients receiving dialysis: A systematic review. Nephrology. 2018;23(8):718-727.

3. Dong R, Guo Z-Y, Ding J-R, Zhou Y-Y, Wu H. Gastrointestinal symptoms: a comparison between patients undergoing peritoneal dialysis and hemodialysis. World J Gastroenterol. 2014;20(32):11370-11375.

4. Zhang J, Huang C, Li Y, et al. Health-related quality of life in dialysis patients with constipation: a cross-sectional study. Patient preference and adherence. 2013;7:589-594.

5. Lehnhardt A, Kemper MJ. Pathogenesis, diagnosis and management of hyperkalemia. Pediatric Nephrology (Berlin, Germany). 2011;26(3):377-384.

6. Gokal R, Alexander S, Ash S, et al. Peritoneal catheters and exit-site practices toward optimum peritoneal access: 1998 update. (Official report from the International Society for Peritoneal Dialysis). Perit Dial Int. 1998;18(1):11-33.

7. Leung L, Riutta T, Kotecha J, Rosser W. Chronic constipation: an evidence-based review. J Am Board Fam Med. 2011;24(4):436-451.

8. Manera KE, Johnson DW, Craig JC, et al. Patient and Caregiver Priorities for Outcomes in Peritoneal Dialysis. Multinational Nominal Group Technique Study. 2019;14(1):74-83.

9. Lee A, Lambert K, Byrne P, Lonergan M. Prevalence of constipation in patietns with advanced kidney disease. Journal of Renal Care. 2016;42(3):144-149.

10. Murtagh FEM, Addington-Hall J, Higginson IJ. The Prevalence of Symptoms in End-Stage Renal Disease: A Systematic Review. Adv Chronic Kidney Dis. 2007;14(1):82-99.

11. Shen $Q$, Zhu H, Jiang G, Liu X. Nurse-Led Self-Management Educational Intervention Improves Symptoms of Patients With Functional Constipation. West J Nurs Res. 2018;40(6):874-888.

12. St-Jules DE, Jagannathan R, Gutekunst L, Kalantar-Zadeh K, Sevick MA. Examining the Proportion of Dietary Phosphorus From Plants, Animals, and Food Additives Excreted in Urine. J Ren Nutr. 2017;27(2):78-83.

13. Naismith DJ, Braschi A. An investigation into the bioaccessibility of potassium in unprocessed fruits and vegetables. Int J Food Sci Nutr. 2008;59(5):438-450.

14. Martínez-Pineda M, Yagüe-Ruiz C, Caverni-Muñoz A, Vercet-Tormo A. Cooking Legumes: A Way for Their Inclusion in the Renal Patient Diet. J Ren Nutr. 2019;29(2):118-125.

15. Kelly JT, Palmer SC, Wai SN, et al. Healthy Dietary Patterns and Risk of Mortality and ESRD in CKD: A Meta-Analysis of Cohort Studies. Clin J Am Soc Nephrol. 2016.

16. Banerjee T, Liu Y, Crews DC. Dietary Patterns and CKD Progression. Blood Purif. 2016;41(13):117-122.

17. Avesani CM, Teta D, Carrero JJ. Liberalizing the diet of patients undergoing dialysis: are we ready? Nephrology Dialysis Transplantation. 2018:gfy309-gfy309.

18. Ghimire S, Castelino RL, Jose MD, Zaidi STR. Medication adherence perspectives in haemodialysis patients: a qualitative study. BMC Nephrol. 2017;18(1):167. 
19. Mechta Nielsen T, Frøjk Juhl M, Feldt-Rasmussen B, Thomsen T. Adherence to medication in patients with chronic kidney disease: a systematic review of qualitative research. Clinical kidney journal. 2018;11(4):513-527.

20. Murali KM, Mullan J, Roodenrys S, Hassan HC, Lambert K, Lonergan M. Strategies to improve dietary, fluid, dialysis or medication adherence in patients with end stage kidney disease on dialysis: A systematic review and meta-analysis of randomized intervention trials. PLoS One. 2019;14(1):e0211479.

21. de Souza RGM, Schincaglia RM, Pimentel GD, Mota JF. Nuts and Human Health Outcomes: A Systematic Review. Nutrients. 2017;9(12):1311.

22. Lamuel-Raventos RM, Onge MS. Prebiotic nut compounds and human microbiota. Crit Rev Food Sci Nutr. 2017;57(14):3154-3163.

23. Liu Z, Lin X, Huang G, Zhang W, Rao P, Ni L. Prebiotic effects of almonds and almond skins on intestinal microbiota in healthy adult humans. Anaerobe. 2014;26:1-6.

24. Barbour JA, Howe PRC, Buckley JD, Bryan J, Coates AM. Nut consumption for vascular health and cognitive function. Nutrition Research Reviews. 2014;27(1):131-158.

25. Bao Y, Han J, Hu FB, et al. Association of Nut Consumption with Total and Cause-Specific Mortality. N Engl J Med. 2013;369(21):2001-2011.

26. Des Jarlais DC, Lyles C, Crepaz N. Improving the reporting quality of nonrandomized evaluations of behavioral and public health interventions: the TREND statement. Am J Public Health. 2004;94.

27. Hoffmann TC, Glasziou PP, Boutron I, et al. Better reporting of interventions: template for intervention description and replication (TIDieR) checklist and guide. BMJ : British Medical Journal. 2014;348:g1687.

28. AUSNUT 2011-13 - Australian Food Composition Database - Release 1.0. 2014. http://www.foodstandards.gov.au/science/monitoringnutrients/afcd/Pages/fooddetails.asp $\underline{x ? P F K I D=F 006081}$.

29. New South Wales Agency for Clinical Innovation. Therapeutic Diet Specifications for Adult Inpatients In:2019.

30. Lewis SJ, Heaton KW. Stool form scale as a useful guide to intestinal transit time. Scand J Gastroenterol. 1997;32(9):920-924.

31. Murphy EL, Murtagh FE, Carey I, Sheerin NS. Understanding symptoms in patients with advanced chronic kidney disease managed without dialysis: use of a short patient-completed assessment tool. Nephron Clin Pract. 2009;111(1):c74-80.

32. Desbrow B, Bauer J, Blum C, Kandasamy A, McDonald A, Montgomery K. Assessment of nutritional status in hemodialysis patients using patient-generated subjective global assessment. J Ren Nutr. 2005;15(2):211-216.

33. Shim J-S, Oh K, Kim HC. Dietary assessment methods in epidemiologic studies. Epidemiology and health. 2014;36:e2014009-e2014009.

34. Ash S, Campbell, K.L. Bogard, J., Millichamp, A. Nutrition prescription to achieve positive outcomes in chronic kidney disease. Nutrients. 2014;6:416-451.

35. Hojat M, Xu G. A visitor's guide to effect sizes: statistical significance versus practical (clinical) importance of research findings. Advances in health sciences education : theory and practice. 2004;9(3):241-249.

36. Grundy MM-L, Lapsley K, Ellis PR. A review of the impact of processing on nutrient bioaccessibility and digestion of almonds. International Journal of Food Science \& Technology. 2016;51(9):1937-1946.

37. Roberfroid M, Gibson GR, Hoyles L, et al. Prebiotic effects: metabolic and health benefits. $\mathrm{Br}$ J Nutr. 2010;104 Suppl 2:S1-63.

38. Sugizaki CSA, Naves MMV. Potential Prebiotic Properties of Nuts and Edible Seeds and Their Relationship to Obesity. Nutrients. 2018;10(11):1645. 
39. Scherer JS, Combs SA, Brennan F. Sleep Disorders, Restless Legs Syndrome, and Uremic Pruritus: Diagnosis and Treatment of Common Symptoms in Dialysis Patients. Am J Kidney Dis. 2017;69(1):117-128.

40. Kimata N, Fuller DS, Saito A, et al. Pruritus in hemodialysis patients: Results from the Japanese Dialysis Outcomes and Practice Patterns Study (JDOPPS). Hemodialysis international International Symposium on Home Hemodialysis. 2014;18(3):657-667.

41. Gagnon AL, Desai T. Dermatological diseases in patients with chronic kidney disease. Journal of nephropathology. 2013;2(2):104-109.

42. Nachbar F, Korting HC. The role of vitamin E in normal and damaged skin. J Mol Med (Berl). 1995;73(1):7-17.

43. Rusu A. Vitamin E in Hemodialysis Patients, . In: Morales-Gonzalez J, ed. Vitamin E in Health and Disease. InTechOpen; 2018.

44. Li H, Xie L, Yang J, Pang X. Symptom burden amongst patients suffering from end-stage renal disease and receiving dialysis: A literature review. International Journal of Nursing Sciences. 2018;5(4):427-431.

45. McCaffrey N, Kaambwa B, Currow DC, Ratcliffe J. Health-related quality of life measured using the EQ-5D-5L: South Australian population norms. Health and quality of life outcomes. 2016;14(1):133.

46. Palliative Care Outcome Scale (POS). How to Score POS version1 and version 2. 2012; https://pos-pal.org/maix/how-to-report.php and https://pos-pal.org/maix/how-toscore.php Accessed February 1, 2019.

47. Herdman M, Gudex C, Lloyd A, et al. Development and preliminary testing of the new fivelevel version of EQ-5D (EQ-5D-5L). Quality of life research : an international journal of quality of life aspects of treatment, care and rehabilitation. 2011;20(10):1727-1736.

48. Pretorius CJ, McWhinney BC, Sipinkoski B, et al. Reference ranges and biological variation of free and total serum indoxyl- and $\mathrm{p}$-cresyl sulphate measured with a rapid UPLC fluorescence detection method. Clin Chim Acta. 2013;419:122-126.

49. Nasreddine ZS, Phillips NA, Bedirian V, et al. The Montreal Cognitive Assessment, MoCA: a brief screening tool for mild cognitive impairment. J Am Geriatr Soc. 2005;53(4):695-699.

50. Tholen S, Schmaderer C, Kusmenkov $E$, et al. Variability of cognitive performance during hemodialysis: standardization of cognitive assessment. Dement Geriatr Cogn Disord. 2014;38(1-2):31-38.

51. Lambert K, Mullan, J.,Mansfield, K.,Lonergan, M.,. A comparison of the extent and pattern of cognitive impairment among predialysis, dialysis and transplant patients: a cross sectional study from Australia. Nephrology 2016:Aug 9. doi: 10.1111/nep.12892. [Epub ahead of print].

52. Tiffin-Richards FE, Costa AS, Holschbach B, et al. The Montreal Cognitive Assessment (MoCA) - a sensitive screening instrument for detecting cognitive impairment in chronic hemodialysis patients. PLoS One. 2014;9(10):e106700.

53. Callahan BJ, McMurdie PJ, Rosen MJ, Han AW, Johnson AJ, Holmes SP. DADA2: Highresolution sample inference from Illumina amplicon data. Nature methods. 2016;13(7):581583. 


\section{Supplemental material}

\section{Methods}

3 Secondary outcomes:

$4 \quad$ Overall symptom burden was evaluated using the Patient Outcome Scale- Renal ${ }^{31}$ at

5 baseline, weeks $1-4,7,10$ of the study. Reporting of scores is as per the reporting guidelines

6 of the authors ${ }^{46}$, with a total score for overall symptom burden ranging from 0 to 68

7 (indicative of a high symptom burden). Quality of life was assessed using the EuroQol 5

8 level Quality of Life Assessment tool (EQ-5D-5L) ${ }^{47}$. This tool was completed at baseline,

9 week 4,7 and 10. Assistance to complete these tools due to poor vision was provided by

10 members of the research team when required. Analysis of crude scores was undertaken using

11 the EuroQOL Crosswalk Value Calculator available from https://euroqol.org/eq-5dinstruments/eq-5d-5l-about/valuation-standard-value-sets/crosswalk-index-value-calculator/ utilising the United Kingdom cross walk scores.

Serum total and free concentrations of the uremic toxins, p-Cresyl Sulfate and Indoxyl

Sulfate were analysed by ultra-performance liquid chromatography (UPLC) using a

fluorescence detection method developed by the research team ${ }^{48}$. Serum samples were stored at $-80^{\circ} \mathrm{C}$ and then analysed in a single batch. The free fraction of each toxin was defined as a percentage of total concentration (free serum concentration divided by the total concentration multiplied by 100). These samples were collected at weeks 1,4, and week 10 .

Serum total and free concentrations of the uremic toxins, $\mathrm{p}$-Cresyl Sulfate (pCS) and Indoxyl Sulfate (IS) were analysed by ultra-performance liquid chromatography (UPLC) using a

24 fluorescence detection method developed by the research team ${ }^{48}$. Serum samples were stored at $-80^{\circ} \mathrm{C}$ and then analysed in a single batch. Chromatography was performed with a Waters 
1 Acquity UPLC I class system comprising of a Binary solvent manager, flow through needle

2 autosampler, fluorescence detector and column manager (Milford MA, USA) and an Acquity

3 HSS T3 $1.8 \mu \mathrm{m}(2.1 \times 50 \mathrm{~mm})$ column with an Acquity BEH C18 $1.7 \mu \mathrm{m}$ VanGuard pre-

4 column $(2.1 \times 5 \mathrm{~mm})$. Mobile phase A was $50 \mathrm{mmol} / \mathrm{L}$ ammonium formate $(\mathrm{pH} 5.0)$ and

5 mobile phase B was $100 \%$ acetonitrile. Mobile phase B increased with a linear gradient from

65 to $25 \%$ over $2.1 \mathrm{~min}$, was then maintained isocratically at $70 \%$ for $0.4 \mathrm{~min}$, followed by

$7 \quad 0.5 \mathrm{~min}$ at $99 \%$. The column was re-equilibrated with initial conditions for $0.5 \mathrm{~min}$. The load-

8 ahead facility within the system was enabled to minimize the run time. Injection volume was

$92 \mu \mathrm{L}$ for total IS (tIS) and total $p \mathrm{CS}$ (tpCS) and $5 \mu \mathrm{L}$ for fIS and $\mathrm{f} p \mathrm{CS}$ samples. Column

10 temperature was maintained at $45^{\circ} \mathrm{C}$. We quantified IS, $p$ CS and the internal standard (50 $\mu \mathrm{mol} / \mathrm{L}$ 4-ethylphenol) with timed programmed fluorescence detection monitoring at specific excitation / emission wavelengths (IS: 300/390 nm; pCS: 260/283 nm; 4-ethylphenol: $285 / 310 \mathrm{~nm})$.

$\mathrm{f} p C S$ and fIS were measured directly, without addition of an internal standard, on ultrafiltrates prepared at room temperature from $200 \mu \mathrm{l}$ of serum centrifuged for $10 \mathrm{~min}$ at $13000 \mathrm{rpm}$ with a 30000 MWCO filter (Merck, Kilsyth, Australia). tIS and t $p$ CS were measured after deproteinization of $100 \mu \mathrm{L}$ serum with $300 \mu \mathrm{l}$ of ethanol that contained internal standard $(50 \mu \mathrm{mol} / \mathrm{L}$ 4-ethylphenol). The mixture was vortexed for $1 \mathrm{~min}$, centrifuged for $5 \mathrm{~min}$ at $13000 \mathrm{rpm}$ and poured into a $2.0 \mathrm{~mL}$ tube that contained $200 \mu \mathrm{L} \mathrm{H}_{2} \mathrm{O}$ and $1 \mathrm{~mL}$ dichloromethane. After vortexing for $1 \mathrm{~min}$ and centrifuging for $5 \mathrm{~min}$ at $13000 \mathrm{rpm}, 150 \mu \mathrm{L}$ of the aqueous supernatant was transferred to an injection vial. The free fraction of each toxin was defined as a percentage of total concentration (free serum concentration divided by the 
1 total concentration multiplied by 100 ). These samples were collected at weeks 1,4 , and week

210.

3 The Montreal Cognitive Assessment (MoCA) Tool ${ }^{49}$ was used to screen for cognitive

4 impairment and changes in cognition during the study. This tool is more sensitive at detecting

5 mild to moderate impairment than other tools such as the Mini Mental State Examination ${ }^{50}$.

6 The MoCA also comes in a range of more than thirty language versions and a version for

7 blind participants which we have utilised in our unit previously ${ }^{51}$. The MoCA was completed

8 at week 1, week 4, and week 10 using a different version of the tool each time to limit

9 learning effects. Assistance to complete these tools due to poor vision was provided by members of the research team when required. A score of $\leq 24 / 30$ was used to indicate cognitive impairment was present ${ }^{52}$.

Faecal samples for gut microbiome analysis were collected from participants using

DNA/RNA shield - Faecal collection tubes (Zymo Research). These samples were collected at baseline, and the commencement of week 4, week 7 and week 10. Samples were frozen at 80C and then analysed in one batch. Briefly, DNA was extracted using a DNeasy PowerSoil HTP 96 DNA Isolation kit (Qiagen)_and the 16S rRNA V4 region was used to study the bacterial community. Amplicon sequencing of the V4 hypervariable region of the bacterial 16S rRNA gene was performed using an Illumina MiSeq platform. The forward and reverse primers for the V4 region were GTGCCAGCMGCCGCGGTAA (5' end 515F) and GGACTACHVGGGTWTCTAAT (3' end 806R), respectively. were first demultiplexed and quality filtered using the q2-demux plugin. This was followed 
1 by denoising with DADA2 ${ }^{53}$ for quality filtering and identification of sequence variants.

2 Sequence variants were aligned and used to construct a phylogeny with FastTree programme

3 (using the q2-phylogeny plugin). Core diversity metrics including alpha-diversity (observed

4 OTUs, Faith's Phylogenetic Diversity), beta-diversity (weighted and unweighted UniFrac)

5 and principal coordinate analysis (PCoA) was calculated at a depth of 5508 sequences per

6 sample. Sequence variants were taxonomically classified against the Silva 132 99\% OTUs

7 reference sequences.

8

\section{Confounders}

10 Demographic and clinical information was collected from the electronic medical record and

11 dialysis flow sheets. Information included age, gender, urine output, laxative use, antibiotic

12 use, phosphate binder type and dose, potassium binder (Resonium) use, dialysis prescription

13 including dialyser size and bath strength, and interdialytic weight gain. A weekly pooled

$14 \mathrm{Kt} / \mathrm{V}$ was also conducted on each patient. Additional pathology measures were taken on a

15 weekly basis including urea, creatinine; bicarbonate; calcium; corrected calcium; magnesium;

16 albumin; C Reactive Protein, liver function tests; and Parathyroid hormone (PTH). 
Table 1. Baseline characteristics of study participants $(n=20)$

\begin{tabular}{|c|c|}
\hline Characteristic & Number \\
\hline Gender (male) & $11(51)$ \\
\hline Age, years median & 67.5 (IQR: 57.5-77.75) \\
\hline Age category, years & \\
\hline $18-54$ & $3(15 \%)$ \\
\hline $55-64$ & $6(30 \%)$ \\
\hline $65-74$ & $7(35 \%)$ \\
\hline $75-84$ & $2(10 \%)$ \\
\hline$>85$ & $2(10 \%)$ \\
\hline Dry weight $(\mathrm{kg})$ & $78(67.1-99.9)$ \\
\hline Nutritional status & \\
\hline Well nourished & $18(90 \%)$ \\
\hline Moderately malnourished & $2(10 \%)$ \\
\hline Severely malnourished & $0(0)$ \\
\hline Anuric & $9(45 \%)$ \\
\hline Comorbidities & \\
\hline Diabetes & $8(40 \%)$ \\
\hline Cerebrovascular Disease & $3(15 \%)$ \\
\hline Peripheral Vascular Disease & $7(35 \%)$ \\
\hline Coronary Artery Disease & $8(40 \%)$ \\
\hline Chronic Lung Disease & $5(25 \%)$ \\
\hline Cognitive impairment present & $9(45 \%)$ \\
\hline MoCA score & \\
\hline$>24 / 30$ (cognitively normal) & $11(55 \%)$ \\
\hline Between 20-24 (mild-moderate impairment) & $8(40 \%)$ \\
\hline Less than $20 / 30$ (severely impaired) & $1(5 \%)$ \\
\hline Laxative use according to type & \\
\hline Nil & $7(35 \%)$ \\
\hline Movicol & $5(25 \%)$ \\
\hline Coloxyl & $7(35 \%)$ \\
\hline Other & $1(5 \%)$ \\
\hline Dietary intake & \\
\hline Protein (g/kg) (Ideal: $1.1 \mathrm{~g} / \mathrm{kg})$ & 1.05 \\
\hline Protein (g/day) & $82.3(21.7)$ \\
\hline Energy $(\mathrm{kJ} / \mathrm{kg}$ ) (Ideal: $125-146 \mathrm{~kJ} / \mathrm{kg}$ ) & 81.5 \\
\hline Energy $(\mathrm{kJ} /$ day $)$ & $6354(1922)$ \\
\hline Sodium (mmol/day) (Ideal: $<100 \mathrm{mmol} /$ day) & $79.4(28.7)$ \\
\hline Sodium (mg/day) & $1826(661)$ \\
\hline Potassium $\mathrm{mmol} / \mathrm{kg}$ (Ideal: $1 \mathrm{mmol} / \mathrm{kg}$ ) & 0.76 \\
\hline Potassium (mg/day) & $2301(671)$ \\
\hline Phosphate (mg/day) & $1221(423)$ \\
\hline Fibre (g/day) (Ideal: $>25 \mathrm{~g} /$ day) & $19.1(7.8)$ \\
\hline Proportion with adequate fibre intake, $\mathrm{n}(\%)$ & $3(15)$ \\
\hline Dialysis adequacy $(\mathrm{Kt} / \mathrm{V})($ mean, $\mathrm{sd})$ & $1.5(0.3)$ \\
\hline
\end{tabular}


Legend: Data are mean (SD), median (interquartile range) or n (\%). MoCA: Montreal Cognitive Assessment (MoCA) Tool. Cognitive Impairment suggested when score <24/30. 
Table 2. Analysis of primary outcomes : safety and efficacy

\begin{tabular}{|c|c|c|c|c|c|c|c|c|}
\hline & Baseline & Week 1 & Week 2 & Week 3 & Week 4 & Week 7 & Week 10 & $\mathrm{P}$ value \\
\hline \multicolumn{9}{|l|}{ Safety outcomes } \\
\hline $\begin{array}{l}\text { Predialysis potassium } \\
(\mathrm{mmol} / \mathrm{L})\end{array}$ & $5.43 \pm 0.66$ & $5.4 \pm 0.6$ & $5.37 \pm 0.63$ & $5.34 \pm 0.69$ & $5.45 \pm 0.69$ & $5.15 \pm 0.96$ & $5.25 \pm 0.66$ & 0.21 \\
\hline Hyperkalemic n (\%) & $6(30)$ & $3(15)$ & $4(21.1)$ & $4(20)$ & $4(20)$ & $4(20)$ & $4(20)$ & 0.96 \\
\hline $\begin{array}{l}\text { Predialysis phosphate } \\
(\mathrm{mg} / \mathrm{dL}) \\
(\mathrm{mmol} / \mathrm{L})\end{array}$ & $\begin{array}{l}4.77 \pm 1.64 \\
1.54 \pm 0.53\end{array}$ & $\begin{array}{l}4.90 \pm 1.58 \\
1.58 \pm 0.51\end{array}$ & $\begin{array}{c}5.33 \pm 1.64 \\
1.72 \pm 0.53\end{array}$ & $\begin{array}{c}5.39 \pm 2.02 \\
1.74 \pm 0.65\end{array}$ & $\begin{array}{c}5.27 \pm 1.89 \\
1.70 \pm 0.61\end{array}$ & $\begin{array}{c}4.37 \pm 1.61 \\
1.41 \pm 0.52\end{array}$ & $\begin{array}{c}4.90 \pm 1.64 \\
1.58 \pm 0.53\end{array}$ & 0.16 \\
\hline Hyperphosphatemic n (\%) & $5(25)$ & $3(15)$ & $6(31.5)$ & $6(30)$ & $9(45)$ & $3(15)$ & $5(25)$ & 0.37 \\
\hline \multicolumn{9}{|l|}{ Efficacy outcomes } \\
\hline $\begin{array}{l}\text { Constipation rated using } \\
\text { Bristol Stool Form Scale, n (\%) }\end{array}$ & $10(50.0)$ & $5(25)$ & $3(16.6)^{*}$ & $1(5.3)^{*}$ & $4(20)$ & $4(22.2)$ & $2(13.3)^{*}$ & $\mathrm{a}$ \\
\hline $\begin{array}{l}\text { Constipation according to } \\
\text { POS-Renal }\end{array}$ & $1.0 \pm 1.0$ & $0.8 \pm 1.1$ & $0.5 \pm 0.8$ & $0.5 \pm 0.8$ & $0.5 \pm 0.7$ & $0.6 \pm 0.8$ & $0.4 \pm 0.7$ & 0.02 \\
\hline $\begin{array}{l}\text { Frequency of bowel movement } \\
\mathrm{n}(\%) \\
\text { Daily } \\
\text { Second daily } \\
<3 \text { times per week }\end{array}$ & $\begin{array}{l}15(75) \\
4(20) \\
1(5)\end{array}$ & $\begin{array}{l}12(63.2) \\
5(26.3) \\
2(10.5)\end{array}$ & $\begin{array}{c}15(78.9) * \\
4(21.1) \\
0\end{array}$ & $\begin{array}{c}15(78.9) * \\
3(15.8) \\
1(5.3)\end{array}$ & $\begin{array}{c}15(78.9)^{*} \\
2(11.1) \\
2(11.1)\end{array}$ & $\begin{array}{c}10(52.6) \\
9(47.3) \\
0\end{array}$ & $\begin{array}{c}12(75.0) \\
3(18.75) \\
1(6.25)\end{array}$ & $\mathrm{b}$ \\
\hline $\begin{array}{l}\text { Frequency of straining } \mathrm{n}(\%) \\
\text { Never/ rarely } \\
\text { Sometimes } \\
\text { Often } \\
\text { Always }\end{array}$ & $\begin{array}{c}9(45) \\
10(50) \\
1(5) \\
0\end{array}$ & $\begin{array}{c}13(68.4) \\
4(21.1) \\
2(10.5) \\
0\end{array}$ & $\begin{array}{c}13(68.4) \\
1(5.3) \\
3(15.8) \\
2(10.5)\end{array}$ & $\begin{array}{c}15(78.9) \\
3(15.8) \\
1(5.3) \\
0\end{array}$ & $\begin{array}{l}11(57.9) \\
6(31.5) \\
1(5.2) \\
1(5.2)\end{array}$ & $\begin{array}{l}9(47.3) \\
7(36.8) \\
1(5.2) \\
3(15.6)\end{array}$ & $\begin{array}{c}12(75.0) \\
3(18.75) \\
1(6.25) \\
0\end{array}$ & - \\
\hline $\begin{array}{l}\text { Frequency of hard/lumpy stool } \\
\text { n }(\%) \\
\text { Never/ rarely } \\
\text { Sometimes }\end{array}$ & $\begin{array}{l}10(50) \\
7(35)\end{array}$ & $\begin{array}{c}10(52.6) \\
7(36.8)\end{array}$ & $\begin{array}{c}12(63.2) \\
5(26.3)\end{array}$ & $\begin{array}{c}14(73.6) \\
4(21.1)\end{array}$ & $\begin{array}{l}11(57.9) \\
6(31.6)\end{array}$ & $\begin{array}{l}9(47.3) \\
9(47.3)\end{array}$ & $\begin{array}{l}10(62.5) \\
3(18.75)\end{array}$ & - \\
\hline
\end{tabular}




\begin{tabular}{|c|c|c|c|c|c|c|c|c|}
\hline $\begin{array}{l}\text { Often } \\
\text { Always }\end{array}$ & $\begin{array}{c}3(15) \\
0\end{array}$ & $\begin{array}{c}2(10.5) \\
0\end{array}$ & $\begin{array}{l}1(5.3) \\
1(5.3)\end{array}$ & $\begin{array}{c}1(5.3) \\
0\end{array}$ & $\begin{array}{c}2(10.5) \\
0\end{array}$ & $\begin{array}{c}1(5.2) \\
0\end{array}$ & $\begin{array}{l}2(12.5) \\
1(6.25)\end{array}$ & \\
\hline $\begin{array}{l}\text { Frequency of incomplete } \\
\text { evacuation } \mathrm{n}(\%) \\
\text { Never/ rarely } \\
\text { Sometimes } \\
\text { Often } \\
\text { Always }\end{array}$ & $\begin{array}{c}8(40) \\
7(35) \\
5(25) \\
0\end{array}$ & $\begin{array}{c}10(52.6) \\
6(31.5) \\
2(10.5) \\
1(5.3)\end{array}$ & $\begin{array}{c}11(57.9) \\
6(31.5) \\
1(5.3) \\
1(5.3)\end{array}$ & $\begin{array}{c}11(61.1) \\
7(38.9) \\
1(5.5) \\
0\end{array}$ & $\begin{array}{c}12(63.2) \\
6(31.6) \\
1(5.3) \\
0\end{array}$ & $\begin{array}{c}10(55.6) \\
8(44.4) \\
1(5.6) \\
0\end{array}$ & $\begin{array}{c}10(62.5) \\
4(25) \\
1(6.25) \\
1(6.25)\end{array}$ & - \\
\hline $\begin{array}{l}\text { Frequency of sensation that } \\
\text { stool cannot pass n }(\%) \\
\text { Never/ rarely } \\
\text { Sometimes } \\
\text { Often } \\
\text { Always }\end{array}$ & $\begin{array}{c}6(30) \\
12(60) \\
2(10) \\
0 \\
\end{array}$ & $\begin{array}{c}14(73.7) \\
3(15.8) \\
1(5.3) \\
1(5.3) \\
\end{array}$ & $\begin{array}{c}14(73.7) \\
5(26.3) \\
0 \\
0 \\
\end{array}$ & $\begin{array}{c}13(61.1) \\
5(27.8) \\
1(5.5) \\
0 \\
\end{array}$ & $\begin{array}{c}13(68.4) \\
6(31.6) \\
0 \\
0 \\
\end{array}$ & $\begin{array}{c}9(50.0) \\
8(44.4) \\
1(5.6) \\
0 \\
\end{array}$ & $\begin{array}{c}10(62.5) \\
5(31.25) \\
1(6.25) \\
0 \\
\end{array}$ & - \\
\hline Laxative use $\mathrm{n}(\%)$ & $13(65)$ & $4(21.1)$ & $4(21.1)$ & $5(26.3)$ & $5(20)^{*}$ & $6(33.3)$ & $6(37.5)$ & $\mathrm{c}$ \\
\hline $\begin{array}{l}\text { Consumption of intervention } \\
\text { (g) }\end{array}$ & - & $\begin{array}{l}36.7 \mathrm{~g} \pm \\
4.03\end{array}$ & $\begin{array}{l}36.7 \mathrm{~g} \\
\pm 5.06\end{array}$ & $\begin{array}{l}34.6 \mathrm{~g} \\
\pm 9.24\end{array}$ & $\begin{array}{c}34.7 \\
\pm 11.9\end{array}$ & - & - & - \\
\hline $\begin{array}{l}\text { Adherence rate to intervention } \\
\% \text { of } 40 \mathrm{~g} \text { consumed per day }\end{array}$ & - & 91 & 92 & 86 & 87 & - & - & - \\
\hline
\end{tabular}

Legend: Reported as mean (sd) or counts (percentage). Serum potassium reported after controlling for dietary potassium intake, serum bicarbonate level, urine output and dialysis adequacy. Serum phosphate reported after dietary phosphate, serum PTH level, urine output and dialysis adequacy. $*=$ indicates $p<0.05$ compared to baseline.

a: Bowel habit assessed using Bristol Stool Form Scale : Compared to baseline: week 2, p=0.04; week 3: p=0.04; Week 10 p=0.04.

b: Frequency of daily bowel movement compared to baseline: week 2: $p=0.03$; week 3: $p=0.03$; week 4: $p=0.04$

c: Proportion using laxatives compared to baseline: week $4: \mathrm{p}=0.02$ 
Table 3. Secondary outcomes

\begin{tabular}{|c|c|c|c|c|c|}
\hline & Baseline & Week 4 & Week 7 & Week 10 & $\mathrm{P}$ value \\
\hline EQ5D5L index score & ${ }^{\mathrm{a}} 0.60(0.51-0.74)$ & ${ }^{\mathrm{a}} 0.72(0.50-0.87)$ & - & ${ }^{\mathrm{a}} 0.75(0.50-0.86)$ & 0.03 \\
\hline EQ5D5L VAS score (range $0-100$ ) & $62.3 \pm 20.2$ & $69.2 \pm 22.7$ & $67.8 \pm 20.9$ & $59.8 \pm 24.1$ & 0.27 \\
\hline PCS (total, umol/ L) (NR: 0.0-38.4) & $167.6 \pm 100.7$ & $176.4 \pm 103.9$ & - & $155.4 \pm 105.1$ & 0.40 \\
\hline PCS (free, umol/ L) (NR:0.1-2.4) & $14.4 \pm 14.8$ & $17.6 \pm 14.3$ & - & $23.7 \pm 18.9$ & 0.18 \\
\hline IS (total, umol/L) (NR: 0.7-6.3) & $136.7 \pm 56.9$ & $133.3 \pm 54.8$ & - & $111.2 \pm 37.9$ & 0.97 \\
\hline IS (free, umol/L) (NR:0.0-0.2) & $25.6 \pm 18.4$ & $32.3 \pm 20.2$ & - & $38.1 \pm 20.4$ & 0.10 \\
\hline MoCA score (maximum score 30 ) & $24.3 \pm 4.3$ & $25.2 \pm 3.5$ & - & $23.1 \pm 5.1$ & 0.05 \\
\hline POS-S Renal total score & $16.7 \pm 6.7$ & $10.2 \pm 6.0$ & $11.7 \pm 6.6$ & $11.3 \pm 9.0$ & 0.002 \\
\hline \multicolumn{6}{|l|}{ POS-S Renal subscores } \\
\hline Pain & $1.1 \pm 1.2$ & $0.6 \pm 1.0$ & $0.8 \pm 1.0$ & $1.2 \pm 1.1$ & 0.22 \\
\hline Shortness of breath & $1.2 \pm 1.3$ & $0.9 \pm 1.2$ & $1.1 \pm 1.2$ & $0.9 \pm 1.2$ & 0.50 \\
\hline Weakness & $1.8 \pm 1.2$ & $1.2 \pm 1.2$ & $1.4 \pm 1.2$ & $1.4 \pm 1.3$ & 0.29 \\
\hline Nausea & $0.8 \pm 0.9$ & $0.5 \pm 0.8$ & $0.4 \pm 0.8$ & $0.7 \pm 0.9$ & 0.25 \\
\hline Vomiting & $0.6 \pm 0.8$ & $0.2 \pm 0.4$ & $0.2 \pm 0.5$ & $0.4 \pm 0.8$ & 0.02 \\
\hline Poor appetite & $0.5 \pm 0.9$ & $0.2 \pm 0.4$ & $0.4 \pm 0.7$ & $0.3 \pm 0.6$ & 0.55 \\
\hline Constipation & $1.0 \pm 1.0$ & $0.5 \pm 0.7$ & $0.7 \pm 0.9$ & $0.4 \pm 0.7$ & 0.02 \\
\hline Mouth problems & $0.3 \pm 0.7$ & $0.3 \pm 0.6$ & $0.5 \pm 0.8$ & $0.5 \pm 1.0$ & 0.57 \\
\hline Drowsiness & $1.2 \pm 1.2$ & $1.0 \pm 1.0$ & $1.2 \pm 1.1$ & $0.9 \pm 1.0$ & 0.70 \\
\hline Poor mobility & $1.2 \pm 1.1$ & $1.0 \pm 1.1$ & $1.0 \pm 0.9$ & $0.8 \pm 1.1$ & 0.49 \\
\hline Itching & $1.3 \pm 1.0$ & $0.7 \pm 0.7$ & $0.7 \pm 0.9$ & $0.9 \pm 1.0$ & 0.006 \\
\hline Difficulty sleeping & $1.4 \pm 1.3$ & $1.0 \pm 1.2$ & $1.2 \pm 1.2$ & $1.0 \pm 1.0$ & 0.42 \\
\hline Restless legs & $1.0 \pm 1.4$ & $0.8 \pm 1.0$ & $0.8 \pm 1.0$ & $0.9 \pm 1.2$ & 0.78 \\
\hline Anxiety & $1.3 \pm 1.4$ & $1.0 \pm 1.4$ & $0.8 \pm 1.1$ & $0.9 \pm 1.1$ & 0.20 \\
\hline Depression & $0.8 \pm 1.2$ & $0.7 \pm 1.2$ & $0.8 \pm 1.2$ & $0.6 \pm 1.1$ & 0.51 \\
\hline Changes in skin & $1.3 \pm 1.4$ & $0.3 \pm 0.6$ & $0.4 \pm 0.7$ & $0.4 \pm 0.7$ & 0.002 \\
\hline Diarrhoea & $0.1 \pm 0.3$ & $0.5 \pm 0.8$ & $0.3 \pm 0.7$ & $0.1 \pm 0.3$ & 0.09 \\
\hline
\end{tabular}

Data reported as mean and standard deviation or ${ }^{\text {a }}$ median and interquartile range 
Legend: NR: normal range; EQ-5D-5L: EuroQol 5 level Quality of Life Assessment tool ; VAS: Visual Analogue Scale; PCS: P Cresyl Sulfate; IS: Indoxyl Sulfate; MoCA: Montreal Cognitive Assessment score; POS-S Renal: Palliative Care Outcome Scale - Renal 
Supplementary Table 1. Individual trend of study participant's predialysis serum potassium over study period (raw data)

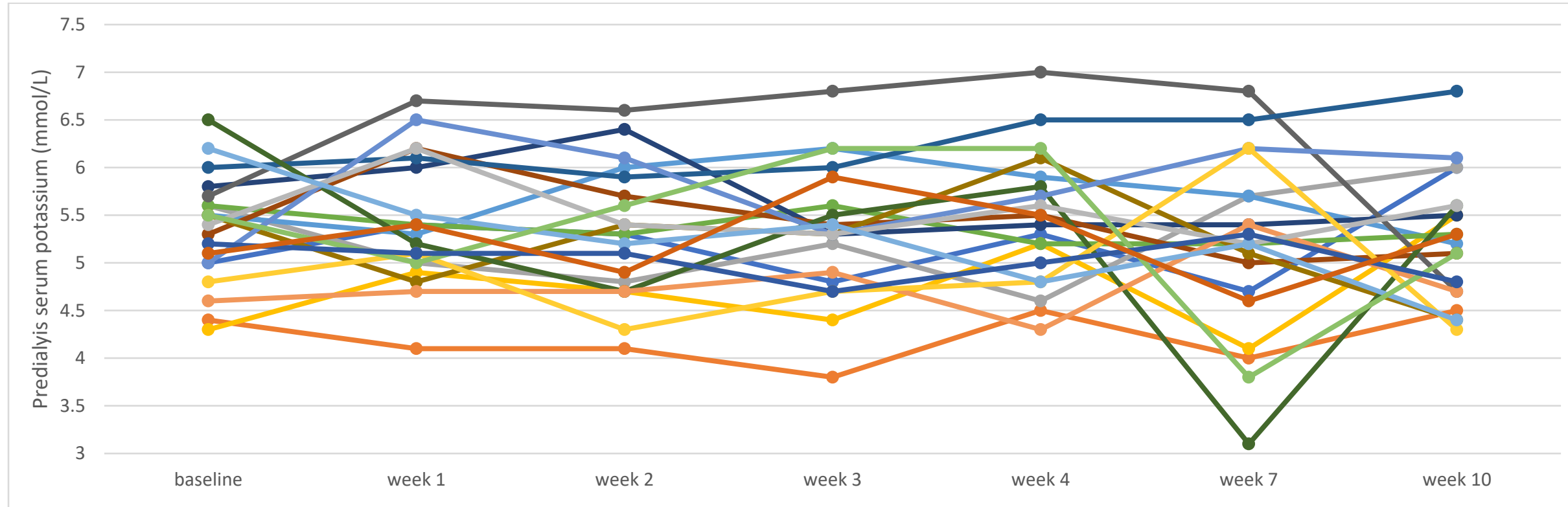


Supplementary Table 2. Individual trend of study participant's predialysis serum phosphate over study period (raw data)

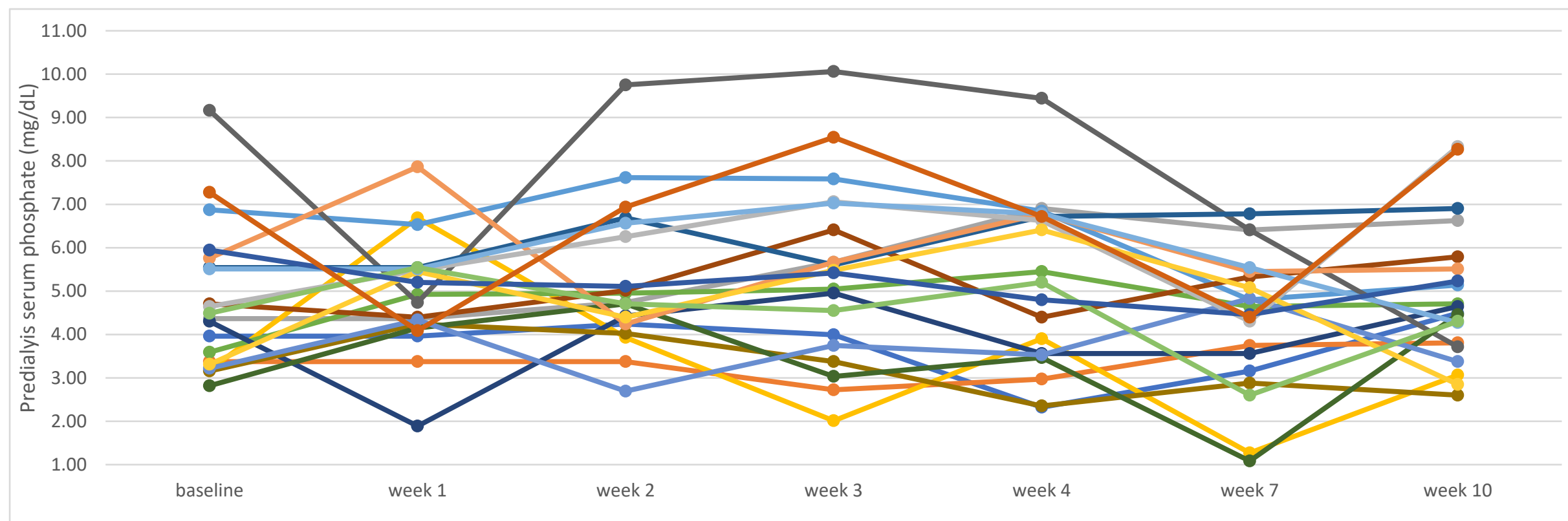

